\section{Untreated illness and outcome of psychosis*}

\author{
MARY CLARKE, PETER WHITTY, STEPHEN BROWNE, ORFHLAITH MCTIGUE, \\ MOAYYAD KAMALI, MAURICE GERVIN, ANTHONY KINSELLA, \\ JOHN L. WADDINGTON, CONAL LARKIN and EADBHARD O'CALLAGHAN
}

\author{
Background There has been \\ controversy as to whether early \\ intervention in psychosis can improve the \\ outcome of the disorder.
}

\begin{abstract}
Aims To establish if there is an association between duration of untreated psychosis and the 4-year outcome of persons with a first episode of psychosis.

Method Prospective naturalistic follow-up study of the outcome of consecutive first presentations with DSM-IV psychosis attending a community-based psychiatric service.
\end{abstract}

Results A longer duration of untreated psychosis was associated with a significantly poorer functional and symptomatic outcome 4 years later. For schizophrenia and schizophreniform disorder, each increment in duration of untreated psychosis was associated with a 7.8 point decrease in global functioning and an increase in positive symptoms scores by 1.9 points.

Conclusions This study extends the findings of short-term follow-up studies by confirming an association between duration of untreated psychosis and 'midterm' outcome.

\section{Declaration of interest None.}

Funded by the Stanley Medical Research Institute.

* Poster presentation at the I3th Biennial Winter Workshop on Schizophrenia, Davos, Switzerland, February 2004.
Although early intervention is well established in numerous branches of medicine, the extent to which this might extend to psychotic illness has engendered considerable controversy. The uncertainty surrounding this issue may reflect the inconsistent findings of studies and also the question whether the link demonstrated between duration of untreated psychosis and short-term outcome is sustained in the long term (Carbone et al, 1999; Robinson et al, 1999; Marshall \& Lockwood, 2004). Inconsistencies between studies may be explained by methodological artefacts such as sampling differences, diagnostic heterogeneity and varying concepts of outcome. In this study we address these issues by following up a representative sample of individuals with a first episode of psychosis, and assessing whether the duration of untreated psychosis is independently associated with pathological and functional outcome 4 years after presentation.

\section{METHOD}

\section{Population}

The sample consisted of the 171 persons resident in a South Dublin urban catchment area of 165000 who, between 1995 and 1999, had experienced a first psychotic episode and had presented either to Cluain Mhuire Family Centre, which provides the public psychiatric service to the catchment area, or to St John of God Hospital, which provides care to persons from the catchment area and elsewhere. First-episode psychosis was defined as first lifetime presentation to any psychiatric service with a psychotic episode, including mania, among all adolescents (12 years of age or over) and adults (no upper age limit). If neuroleptic medication had been prescribed before presentation, for example by a family practitioner, patients were incepted provided they had not been receiving medication for more than 30 days before referral to the service (Browne et al, 2000). At the time of the study there were no early detection strategies in place. Following approval by the Research Ethics Committee of St John of God Hospital, all those approached gave verbal consent to their participation in the study.

\section{Assessments}

At presentation, we assessed psychopathology using the Positive and Negative Syndrome Scale (PANSS; Kay et al, 1986) and global functioning using the Global Assessment of Function scale (GAF; First et al, 1995). When clinically stable, we used the Structured Clinical Interview for DSM (SCID) to ascertain DSM-IV Axis I diagnoses (American Psychiatric Association, 1994); socio-demographic information was also recorded. During the course of the SCID I interview, participants dated, to the nearest month, their first experience of the onset of psychotic symptom(s); they detailed all previous contacts with the health services, police and judiciary, and any treatment received during the prodromal and/or psychotic phases. Their consent was then sought to interview their parent(s) or nearest (geographically) living adult relative. Where possible, we requested that either both parents or at least two family members should attend. In this interview, supplementary information on premorbid, prodromal and onset phases of the illness was collected. We assessed functioning over the premorbid period using the Premorbid Social Adjustment (PSA) scale (Foerster et $a l, 1991)$, which addresses the patient's premorbid functioning over two distinct time periods: between 5 and 11 years of age (PSA1) and between 12 and 16 years of age (PSA2). To increase the validity of premorbid data, we used only objective measures of premorbid functioning given by a parent or an older relative with an adequate knowledge of the patient during this time. Furthermore, as some participants experienced the onset of psychotic or prodromal symptoms during the PSA2 time period, we used only PSA1 scores in analyses.

In the family interview, we evaluated the emergence of different phases of illness using the scale of Beiser and colleagues (Beiser et al, 1993). Duration of untreated psychosis was defined as the interval between first noted psychotic symptom(s) and presentation to the psychiatric services for initiation of adequate treatment of a 
psychotic illness; first manic symptom(s) were used for bipolar disorder. This figure was based on independent parental or relative and participant interviews; in instances where there was discrepancy between these two figures, or where there was no information available from a relative, we assigned a consensus duration using all available sources of information. Onset of the prodrome was defined as earliest noted deviation from the person's premorbid functioning or emergence of prodromal symptoms as defined in the Beiser scale sections: attitude/thinking, mood, behaviour/ performance and somatic symptoms. The duration of prodrome was defined as time between onset of prodromal symptoms and the onset of first psychotic symptom(s); this value, when added to duration of untreated psychosis, gave duration of untreated illness.

At 4 years following inception, all participants were contacted and invited to return for follow-up assessment. During these interviews, psychopathology and global functioning were assessed as above by an investigator masked to initial scores and to duration of untreated psychosis.

\section{Statistical analysis}

For outcome analyses, remission was defined as no score higher than 3 over the previous month on any PANSS item at follow-up (Larsen et al, 2000). Paired $t$-tests were used to compare scores at presentation with those at 4-year follow-up. We examined the relationship between variables (duration of untreated psychosis, prodrome, gender, years in education, age at onset of psychotic symptoms, PSA1 score) and outcome dimensions using correlation analyses. We then used stepwise multiple logistic and linear regression procedures to ascertain whether duration of untreated psychosis was associated with outcome independent of other significant variables. In these analyses, we controlled for scores at presentation and factors known to influence duration of untreated psychosis at presentation (diagnosis and social withdrawal). The relationship between duration measured as a binary variable, split at the median score, and other variables was assessed using Cohen's effect sizes (Cohen, 1969). We repeated the outcome analyses confining the group to those with a diagnosis of schizophrenia or schizophreniform disorder.
We evaluated interrater reliabilities for the PANSS by the concurrent examination of 10 patients with chronic schizophrenia. We rated family interviews in patients with first-episode psychosis to assess interrater reliabilities for the PSA and the dating of the onset of psychotic symptoms.

\section{RESULTS}

\section{Interrater reliabilities}

The intraclass correlation coefficients between raters were as follows: PANSS positive subscale $(r=0.94)$, PANSS negative subscale $(r=0.73)$, PANSS total score $(r=0.88)$, PSA2 $(r=0.92)$, duration of untreated psychosis $(r=0.94)$.

\section{Participants' characteristics at presentation}

The sample consisted of 171 consecutive presentations to the clinical services with a first episode of psychosis. All patients completed initial clinical assessment (PANSS and SCID), but 22 refused consent to contact a relative. Participants were predominantly male $(99,58 \%)$, single $(146$, $85.4 \%$ ) and living in the family home $(137,80.1 \%)$. The mean age at presentation was 28.5 years (s.d.=11.1). At presentation, $101(59.1 \%)$ met DSM-IV criteria for schizophrenia or schizophreniform disorder, 13 (7.6\%) for delusional disorder, 25 (14.6\%) for bipolar disorder on the basis of a first episode of manic symptoms, $11(6.4 \%)$ for major depressive disorder with a first episode of psychotic features, $12(7.0 \%)$ for substance-induced psychosis, $5(2.9 \%)$ for psychosis owing to a general medical condition and 4 $(2.3 \%)$ psychosis not otherwise specified. No cases of schizoaffective disorder or brief psychotic disorder were encountered. The majority, $144(84.2 \%)$ were treated initially as in-patients. For subsequent analyses, patients with a diagnosis of psychosis owing to a general medical condition were excluded, leaving 166 participants.

Premorbid adjustment scores were unavailable for 38 of the total group (parents deceased or permission to contact them refused) including 11 of the group with schizophrenia.

\section{Duration of untreated psychosis at presentation}

For the 166 participants, mean duration was 17.9 months $\quad$ (s.d. $=32.1$, range
0.25-240, median 5 months, interquartile range (IQR) 1, 24); mean prodrome, available for 152 participants, was 21.3 months (s.d. $=35.7$, range $0-177$, median 3 months, IQR 0, 31).

\section{Follow-up at 4 years}

Of the 166 participants, 132 (79.5\%) consented to a second direct interview and assessment 4 years after presentation; 4 $(2.4 \%)$ had died by suicide and 30 $(18.1 \%)$ refused follow-up assessment or were untraceable. There were no significant differences between those who participated in follow-up and those who did not in terms of duration of untreated psychosis, length of prodrome, years in education, premorbid social adjustment, gender, age, psychopathology or global functioning at presentation, either within the overall group or among those with a diagnosis of schizophrenia or schizophreniform disorder.

PANSS data were available for 129 of the 132 participants and, of these, 76 (57.6\% of 132 ) had no score higher than 3 for any PANSS item at 4-year follow-up and were therefore defined as in remission (Larsen et al, 2000) (see Method). Global functioning and total, positive and negative PANSS psychopathology all materially improved between presentation and 4-year follow-up (Table 1).

\section{Remission at 4-year follow-up}

A stepwise multiple logistic regression model $\left(\chi^{2}=7.3, P<0.01\right)$, including gender, diagnosis, age at onset, years in education, PSA1 score, duration of untreated psychosis, prodrome and social withdrawal indicated that remission was associated with only gender (female) $(\beta=1.27$, s.e. $=0.5$, $P=0.01 ; \operatorname{Exp} \beta=3.5,95 \%$ CI 1.3 to 9.3 ) with a trend effect for a shorter duration, $P=0.08$. Among the 82 participants with schizophrenia or schizophreniform disorder $53.7 \%$ were in remission. Stepwise multiple regression $\left(\chi^{2}=6.5, P=0.01\right)$, including the same variables apart from diagnosis, indicated that remission was associated with a shorter duration $(\beta=0.37, P=0.02$; $\operatorname{Exp}$ $\beta=1.4,95 \%$ CI 1.08 to 1.93 ), with a trend effect for a shorter prodrome, $P=0.07$ and female gender, $P=0.07$.

\section{Global functioning at 4-year follow-up}

For the 132 participants, lower GAF score at 4-year follow-up (GAF4) was associated 
Table I Comparison of scores at presentation and 4-year follow-up

\begin{tabular}{lccccc}
\hline Variable & $\begin{array}{c}\text { Score at } \\
\text { presentation }\end{array}$ & $\begin{array}{c}\text { Score at 4-year } \\
\text { follow-up }\end{array}$ & $\begin{array}{c}\text { Mean } \\
\text { difference }\end{array}$ & $P$ & $95 \% \mathrm{Cl}$ \\
\hline GAF $(n=132)$ & 22.9 & 62.3 & 39.4 & $<0.001$ & $36.1,42.6$ \\
PANSS-TOT $(n=129)$ & 74.4 & 48.6 & -25.8 & $<0.001$ & $-29.4,-22.1$ \\
PANSS-POS $(n=129)$ & 21.3 & 10.2 & -11.1 & $<0.001$ & $-12.5,-9.8$ \\
PANSS-NEG $(n=129)$ & 15.7 & 13.0 & -2.7 & $<0.001$ & $-3.9,-1.5$ \\
\hline
\end{tabular}

GAF, global assessment of function score; PANSS, Positive and Negative Syndrome Scale; PANSS-TOT, PANSS total symptom score; PANSS-POS, PANSS positive symptom score; PANSS-NEG, PANSS negative symptom score.

with increasing duration of untreated psychosis $(r=0.35, P<0.001)$, increasing duration of untreated illness $(r=0.35, P=0.001)$ and earlier age at onset of psychosis $(r=0.24, P<0.01$ ), but not with length of prodrome, years in education or PSA1 scores. On confining analysis to those 82 patients with schizophrenia or schizophreniform disorder, lower GAF4 score was associated with increasing duration of untreated psychosis $(r=-0.43, P<0.001)$, increasing duration of untreated illness $(r=-0.33, P=0.003)$, fewer years of education $(r=0.30, P<0.01)$ and earlier age at onset of psychosis $(r=0.27, P<0.01)$, but not with length of prodrome or PSA1 score.

In a stepwise multiple linear regression model $(F=14.8, P<0.001)$, controlling for GAF score at presentation (GAF1), diagnosis, gender, age at onset of psychotic symptoms, social withdrawal at presentation and prodromal length, worse GAF score at 4-year follow-up (GAF4) was associated with longer duration of untreated psychosis, male gender and social withdrawal (Table 2). The unstandardised coefficients indicated that a unit increase in duration (log-transformed) resulted in reduction in GAF4 scores of 5.5. We repeated the analysis substituting duration of untreated illness for duration of untreated psychosis, and found the results practically unchanged. We also examined the relationship of duration of untreated psychosis with GAF4 score by calculating effect sizes. Duration data were divided by median score into short- and long-duration groups. The effect size for duration on GAF4 outcome was -0.59 (moderate); in practice, this implies that $73 \%$ of participants in the short-duration group would have a GAF4 score higher than the mean GAF4 score of the long-duration group. On confining analysis to those participants with schizophrenia or schizophreniform
Table 2 Multiple regression model of 4-year global outcome

\begin{tabular}{lrrrl}
\hline Measure & Adj. $R^{2}$ & \multicolumn{1}{c}{$\beta$} & s.e. & \multicolumn{1}{c}{$P$} \\
\hline \multicolumn{2}{c}{ All diagnoses, $n=129$} & & & \\
Social withdrawal & 17.5 & -3.1 & 0.9 & 0.001 \\
DUP & 4.2 & -5.5 & 2.0 & 0.007 \\
Gender & 2.6 & 6.8 & 2.0 & 0.03 \\
DUI & 4.1 & -5.5 & 2.0 & 0.007
\end{tabular}

Schizophrenia/schizophreniform disorder, $n=82$ $\begin{array}{lllll}\text { DUP } & 17.3 & -7.8 & 2.3 & 0.001\end{array}$ $\begin{array}{lllll}\text { Gender } & 4.6 & 10.4 & 3.5 & 0.004\end{array}$ $\begin{array}{llllll}\text { Educational } & 6.3 & 1.9 & 0.7 & 0.006\end{array}$ $\begin{array}{lllll}\text { DUI } & 10.7 & -7.0 & 2.5 & 0.006\end{array}$

Adj., adjusted; s.e., standard error; DUP, duration of untreated psychosis; DUI, duration of untreated illness (substituted for DUP); GAF, global assessment of function score; GAFI, GAF score at presentation.

disorder, and controlling for gender, age at onset, years in education, scores at presentation and prodrome, stepwise multiple regression $(F=11.6, P<0.001)$ indicated GAF4 score to be associated with duration of untreated psychosis, gender and years in education. GAF4 scores were reduced by 7.8 points for each unit increase in duration. We repeated the analysis substituting duration of untreated illness and found the effect to be weaker but nonetheless significant (Table 2). The effect size for duration of untreated psychosis on GAF4 outcome was -0.65 (moderate), implying that $76 \%$ of people in the short-duration group would have a GAF4 score higher than the mean GAF4 score of the longduration group.

\section{Psychopathology at 4-year follow-up}

Among all 129 participants, higher PANSSpositive score at 4-year follow-up
(PANSS4-POS) was associated with increasing duration of untreated psychoses $(r=0.29, P<0.01)$, increasing duration of untreated illness $(r=0.20, P=0.03)$ and with earlier age at onset $(r=0.23$, $P<0.01$ ), but not with length of prodrome, years in education or PSA1 score; on confining the analysis to those 82 participants with schizophrenia or schizophreniform disorder, higher PANSS4-POS score was associated with increasing duration of psychosis $(r=0.32, P<0.01)$, but not with age at onset, length of prodrome, years in education or PSA1 score.

In a stepwise multiple linear regression model $(F=8.3, P<0.001)$, controlling for PANSS-POS score at presentation (PANSS1-POS), diagnosis, gender, age at onset of psychotic symptoms, prodromal length and social withdrawal, the PANSS4-POS score was associated with gender and duration of untreated psychosis (Table 3). The effect size for duration on PANSS4-POS was 0.58 (moderate), implying that $73 \%$ of people in the shortduration group would have a PANSS4POS outcome score lower than the mean PANSS4-POS score of the long-duration group. When we substituted illness for psychosis, this did not make a significant contribution to the model. On confining analyses to those participants with schizophrenia or schizophreniform disorder, stepwise multiple regression $(F=7.0, P=0.002)$ indicated a stronger effect for psychosis. Each unit increment in duration of untreated psychosis resulted in an increase of 1.9 in PANSS4-POS scores. A repeat model using illness instead of psychosis demonstrated that duration of untreated illness

Table 3 Multiple regression model of 4-year PANSS positive symptom score

\begin{tabular}{lcccc}
\hline Measure & Adj. $R^{2}$ & $\beta$ & s.e. & $P$ \\
\hline All diagnoses, $n=129$ & & & & \\
Gender & 6.6 & -2.2 & 0.78 & 0.005 \\
DUP & 3.8 & 1.2 & 0.50 & 0.02 \\
DUI & - & - & - & NS
\end{tabular}

Schizophrenia/schizophreniform disorder, $n=82$ $\begin{array}{lllll}\text { DUP } & 9.3 & 1.9 & 0.7 & 0.007\end{array}$ $\begin{array}{llllll}\text { Gender } & 4.3 & -2.2 & 1.0 & 0.04\end{array}$ $\begin{array}{lllll}\text { DUI } & 4.3 & 1.6 & 0.73 & 0.03\end{array}$

PANSS, positive and negative syndrome scale; Adj., adjusted; DUP, duration of untreated psychosis; DUI, duration of untreated illness (substituted for DUP); NS, not significant. 
made a smaller but significant contribution to the model (Table 3). The effect size for duration of untreated psychosis on PANSS4-POS outcome was 0.73 (moderate), implying that $76 \%$ of people in the short-duration group would have a PANSS4-POS outcome score lower than the mean PANSS4-POS score of the long-duration group.

Among the 129 participants, higher PANSS-negative score at 4-year follow-up (PANSS4-NEG) was associated with increasing duration of untreated psychosis $(r=0.29, P<0.05)$, increasing duration of untreated illness $(r=0.27, P=0.003)$, fewer years in education $(r=-0.18, P<0.05)$, younger age at onset $(r=-0.19, P<0.05)$ and higher or worse PSA1 score $(r=0.29$, $P<0.01$ ), but not with length of prodrome; on confining analyses to those 82 participants with schizophrenia or schizophreniform disorder, higher PANSS4-NEG score was associated similarly with increasing duration of untreated psychosis $(r=0.32, P<0.01)$, fewer years in education $(r=-0.30, P<0.01)$, younger age at onset $(r=-0.25, P<0.05)$ and higher or worse PSA1 score $(r=0.26, P<0.05)$, but not with length of untreated prodrome $(r=0.07)$.

Stepwise multiple linear regression $(F=17.0, \quad P<0.001)$, including PANSSNEG score at presentation (PANSS1NEG), diagnosis, gender, age at onset of psychotic symptoms, educational years, PSA1, prodromal length and social withdrawal, PANSS4-NEG score was associated with PANSS1-NEG, years in education and duration of untreated psychosis (Table 4); the effect size for duration on PANSS4-NEG was 0.37 (small), implying that $66 \%$ of people in the short-duration group would have a PANSS4-NEG score lower than the mean PANSS4-NEG score of the long-duration group. On confining analyses to those participants with schizophrenia or schizophreniform disorder, stepwise multiple regression $(F=17.0, P<0.001)$ including PANSS1-NEG, gender, years in education, duration of untreated psychosis and prodrome, indicated PANSS4-NEG score to be associated with PANSS1-NEG, years in education and duration (Table 4). The effect size for duration on PANSS4 NEG was 0.35 (small), implying that $66 \%$ of people in the short-duration group would have a PANSS4-NEG score lower than the mean PANSS4-NEG score of the long-duration group. Duration of untreated illness made no independent contribution to the model.
Table 4 Multiple regression model of 4-year PANSS negative symptom score

\begin{tabular}{|c|c|c|c|c|}
\hline Measure & Adj. $R^{2}$ & $\beta$ & s.e. & $P$ \\
\hline \multicolumn{5}{|c|}{ All diagnoses, $n=129$} \\
\hline PANSSI-NEG & 24.3 & 0.39 & 0.07 & 0.00 \\
\hline Educational years & 5.8 & -0.59 & 0.20 & 0.005 \\
\hline DUP & 2.6 & 1.6 & 0.76 & 0.03 \\
\hline DUI & - & - & - & NS \\
\hline \multicolumn{5}{|c|}{ Schizophrenia/schizophreniform disorder, $n=82$} \\
\hline PANSSI-NEG & 22.9 & 0.40 & 0.08 & 0.00 \\
\hline Educational years & 7.1 & -0.74 & 0.25 & 0.004 \\
\hline DUP & 3.2 & -0.6 & 0.28 & 0.04 \\
\hline DUI & - & - & - & NS \\
\hline
\end{tabular}

PANSS, positive and negative syndrome scale; $\mathrm{Adj}$. adjusted; PANSSI-NEG, PANSS negative score at presentation; DUP, duration of untreated psychosis; DUI, duration of untreated illness, substituted for DUP. NS, not significant.

\section{DISCUSSION}

This study concerns a large, prospectively ascertained, epidemiologically complete and hence representative population of people experiencing their first psychotic episode. We analysed the association of duration of untreated psychosis with both functional and psychological outcome over 4-year follow-up across diagnoses. The principal finding was that increasing duration was independently associated with reduced likelihood of remission, poorer functional outcome and increasing psychopathology at 4 years. The issue of whether the effect of duration extends beyond relatively short-term follow-up is critical, given that most (Wiermsa et al, 1998; Robinson et al, 1999; Craig et al, 2000) but not all (Bottlender et al, 2003) longer-term prospective follow-up studies have reported negative findings. Norman \& Malla (2001) have postulated that the predictive power of initial duration of untreated psychosis may weaken over time. However, they suggest that indices of outcome other than remission and relapse should be examined.

\section{Strengths}

One of the strengths of this study is the representativeness of the sample (Friis et al, 2003). This was achieved by sourcing the sample from a geographically defined area, including consecutive out-patients as well as in-patients, and not applying exclusion criteria relating to age or substance misuse. Furthermore, the initial measures were an integral part of a comprehensive clinical assessment and included all cases of psychosis within the catchment area referred for assessment. Although there was a degree of sample attrition during the initial and follow-up assessments, this was less than $30 \%$. All the follow-up interviews were conducted face to face by an interviewer, and did not rely solely on chart information or telephone interviews. The researcher who conducted the follow-up was masked to both duration and inception ratings.

As many of the negative studies have related to samples with schizophrenia only (Robinson et al, 1999; Barnes et al, 2000; Ho et al, 2000), we analysed schizophrenia and schizophreniform disorder separately and found some difference. However, apart from negative symptoms, the effect of duration of untreated psychosis mostly became stronger. Nonetheless, the differences between schizophrenia and schizophreniform disorder and the total group suggest that the results from a mixed first-episode sample may not translate to individual diagnostic categories. We dealt with the skewed duration data and controlled for both confounders, and values of a particular outcome variable at presentation (Norman \& Malla, 2001; Friis et al, 2003). We broadened the outcome variables beyond remission and relapse, particularly as another study has shown that duration may have a selective influence on some aspects of outcome but not others (Malla et al, 2002).

\section{Limitations}

Limitations to the study include the fact that the sample size did not permit examination of individual diagnostic categories other than schizophrenia and schizophreniform disorder, only three dimensions of outcome were assessed and the results are at an associative rather than causative level and did not involve the manipulation of the duration variable. No cases of schizoaffective disorder or brief psychotic disorder were diagnosed at inception. At 4-year follow-up, when the SCID was repeated by an investigator masked to the original diagnosis, three cases of schizophrenia were subsequently diagnosed with schizoaffective disorder (Whitty et al, 2005). Nonetheless, the paucity of these cases at inception remains a cause of concern. 
Another weakness relates to the measurement of the prodrome and its inclusion in the statistical analyses. It is difficult to measure the prodrome reliably, and one could argue that it should be excluded from the data. However, given evolving interest in, but concerns over, the reliable measurement of duration of untreated illness as distinct from untreated psychosis, these are offered as exploratory analyses to stimulate further work in a controversial area.

\section{Level of functioning and negative symptoms}

This study showed that duration of untreated psychosis is associated with global functioning. These findings are broadly consistent with the results from an early intervention project in Norway that showed that the early-detection group had sustained improvement in global functioning but not in positive symptoms at 3-month follow-up (Melle et al, 2004). Thus duration of untreated psychosis may have a selective effect, influencing some dimensions of outcome more than others (Malla et al, 2002).

Global negative symptoms at presentation emerged as the best predictor of the level of negative symptoms at 4 years. However, contrary to other findings (Wiermsa et al, 1998; Craig et al, 2000; Larsen et al, 2000; Malla et al, 2002), we found that duration of untreated psychosis made a small contribution to the model in the total group. Unlike Harrigan et al (2003), we did not find a major influence for duration of untreated psychoses in the schizophrenia and schizophreniform disorder group. Indeed, the direction was opposite to that which we had anticipated. Analysis of the data using effect sizes (which are less influenced by sample size) demonstrated small effect sizes for duration of untreated psychosis in the total group and the schizophrenia and schizophreniform disorder group. Negative symptoms tend to be less responsive to pharmacological intervention, and it remains to be seen whether medication and intensive psychosocial rehabilitation in combination with early intervention will have more of an impact in tackling negative symptoms.

Keshavan et al (2003) have recently highlighted the importance of considering the effect of duration of untreated illness on outcome. In their sample of 101 individuals with a first episode of psychosis, they found that the duration of untreated illness

MARY CLARKE, MD, MRCPI, MRCPsych, PETER WHITTY, MD, MRCPsych, STEPHEN BROWNE, MD, MRCPsych, ORFHLAITH MCTIGUE, MRCPsych, MOAYYAD KAMALI, MRCPsych, MAURICE GERVIN, MRCPsych, Department of Psychiatry, University College Dublin, and Stanley Research Unit, Cluain Mhuire Family Centre, St John of God Adult Psychiatric Service, Dublin; ANTHONY KINSELLA, FIS, Department of Mathematics, Dublin Institute of Technology; JOHN L.WADDINGTON, DSc, Stanley Research Unit, Department of Clinical Pharmacology, Royal College of Surgeons in Ireland, Dublin; CONAL LARKIN, FRCPI, FRCPsych, EADBHARD O'CALLAGHAN, MD, FRCPI, FRCPsych, Department of Psychiatry, University College, Dublin, and Stanley Research Unit, Cluain Mhuire Family Centre, St John of God Adult Psychiatric Service, Dublin, Ireland

Correspondence: Professor Eadbhard O'Callaghan, DELTA/DETECT Early Intervention in Psychosis Services, I Marine Tec., Dun Laoghaire, Co. Dublin, Ireland. Tel: +353 I2I72100; fax: +353 I 2833886; email: eadbhard@gmail.com

(First received 10 June 2005, final revision 7 February 2006, accepted 2 May 2006)

was a stronger predictor of 2-year outcome than that of psychosis. However, these effects were marginal. In this study, the duration of untreated illness was associated with global functioning at 4 years and with positive symptoms in the schizophrenia and schizophreniform disorder group, but had no effect on negative symptoms. The duration of the prodrome was not significantly associated with any of the outcome variables, apart from negative symptoms in the schizophrenia and schizophreniform group. Other studies have found that the prodrome did not influence time to remission or level of remission (Loebel et al, 1992; Malla et al, 2002), negative symptoms (Malla et al, 2002; Harrigan et al, 2003), but had small effects on positive symptoms (Malla et al, 2002) and a significant association with functional outcome (Harrigan et al, 2003). It is possible that the duration of the prodrome phase may have an independent effect on outcome, but our results suggest a more robust duration of untreated psychosis.

\section{Early intervention}

The association between duration of untreated psychosis and functional outcome supports the likely benefits of early intervention in psychosis. Nonetheless, successful reduction of the duration may not be as easy as it sounds. In addition to a specialist rapid-access programme, most early intervention services include an educational component that aims to increase the ability of general practitioners to recognise the early stages of psychosis. However, with the increased availability of counselling practices and alternative medical services, it will be important to find out whether in fact general practitioners are usually the first port of call in the early stages of a psychotic illness. Examining pathways to care available to such individuals may provide these answers. Furthermore, a clearer understanding of the sociocultural factors that influence disease recognition, particularly among family members, may also be helpful.

\section{REFERENCES}

American Psychiatric Association (1994) Diagnostic and Statistical Manual of Mental Disorders (4th edn) (DSM-IV). Washington, DC: APA.

Barnes, T., Hutton, S., Chapman, M., et al (2000) West London first-episode study of schizophrenia: clinical correlates of duration of untreated psychosis. British Journal of Psychiatry, 177, 207-2II.

Beiser, M., Erickson, D., Fleming, J., et al (1993) Establishing the onset of psychotic illness. American Journal of Psychiatry, 158, 1349-1354.

Bottlender, R., Sato, T., Jager, M., et al (2003) The impact of duration of untreated psychosis prior to first admission on the 15 -year outcome in schizophrenia. Schizophrenia Research, 62, 37-44.

Browne, S., Clarke, M., Gervin, M., et al (2000) Determinants of neurological dysfunction in first episode schizophrenia. Psychological Medicine, 30, |433-144|.

Carbone, S., Harrigan, S., McGorry, P. D., et al (1999) Duration of untreated psychosis and 12 month outcome in first episode psychosis: the impact of treatment approach. Acta Psychiatrica Scandinavica, 100, 96-104.

Cohen, J. (1969) Statistical Power Analysis for the Behavioral Sciences. New York: Academic Press.

Craig, T., Bromet, E., Fennig, S., et al (2000) Is there an association between duration of untreated psychosis and 24-month clinical outcome in first admission series? American Journal of Psychiatry, 157, 60-66.

First, M. B., Spitzer, R. L., Gibbon, M., et al (1995) Structured Clinical Interview for DSM-IV Axis I Disorders - Patient Edition (SCID I/P, Version 2.0). New York: New York State Psychiatric Institute.

Foerster, A., Lewis, S., Owen, M., et al (1991) Pre-morbid adjustment and personality in psychosis: effects of sex and diagnosis. British journal of Psychiatry, 158, $|7|-176$.

Friis, S., Larsen, T. K., Melle, I., et al (2003)

Methodological pitfalls in early detection studies - the NAPE Lecture 2002. Acta Psychiatrica Scandinavica, 107 3-9. 
Harrigan, S., McGorry, P. D. \& Krstev, H. (2003) Does treatment delay in first episode psychosis really matter? Psychological Medicine, 33, 97-110.

Ho, B. C., Andreasen, N., Flaum, M., et al (2000) Untreated initial psychosis: its relationship to quality of life and symptom remission in first episode schizophrenia. American Journal of Psychiatry, 157 808-815

Kay, S. R., Opler, L. A. \& Fiszbein, A. (1986) Positive and Negative Syndrome Scale (PANSS) Rating Manual. New York: Department of Psychiatry, Albert Einstein College of Medicine, Montefiore Medical Centre and Schizophrenia Research Unit.

Keshavan, M. S., Haas, G., Miewald, J., et al (2003) Prolonged untreated illness duration from prodromal onset predicts outcome in first episode psychoses. Schizophrenia Bulletin, 29, 757-769.

Larsen, T. K., Moe, L. C.,Vibe-Hansen, L., et a (2000) Premorbid functioning versus duration of untreated psychosis in I year outcome in first episode psychosis. Schizophrenia Research, 45, I-9.

Loebel, A. D., Lieberman, J. A., Alvir, J. M., et al (1992) Duration of psychosis and outcome in firstepisode schizophrenia. American Journal of Psychiatry, 149, 1183-1188.

Malla, A. K., Norman, R. M. G., Manchanda, R., et al (2002) One year outcome in first episode psychosis: influence of DUP and other predictors. Schizophrenia Research, 54, 231-242.

Marshall, M. \& Lockwood, A. (2004) Early Intervention for Psychosis. Cochrane Database of Systematic Reviews, 2: CD0047I8.

Melle, I., Larsen, T. K., Haahr, U., et al (2004) Reducing the duration of untreated first-episode psychosis: effects on clinical presentation. Archives of General Psychiatry, 6I, 143-150.
Norman, R. M. \& Malla, A. K. (200I) Duration of untreated psychosis: a critical examination of the concept and its importance. Psychological Medicine, 31 $381-400$.

Robinson, D., Woerner, M. G., Alvir, J. M., et al (1999)

Predictors of relapse following response from a first episode of schizophrenia or schizoaffective disorder. Archives of General Psychiatry, 56, 24I-247.

Whitty, P., Browne, S., Clarke, M., et al (2005) Systematic comparison of subjective and objective measures of quality of life at 4-year follow-up subsequent to a first episode of psychosis. Journal of Nervous and Mental Disease, 192, 805-809.

Wiermsa, D., Nienhuls, F., Slooff, C. J., et al (1998) Natural course of schizophrenic disorders: a 15 year follow-up of a Dutch incidence cohort. Schizophrenic Bulletin, 24, 75-85. 